\title{
ALMA OBSERVATIONS OF THE GALACTIC CENTER: SiO OUTFLOWS AND HIGH-MASS STAR FORMATION NEAR Sgr A*
}

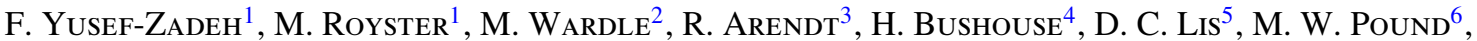 \\ D. A. RoberTs ${ }^{1}$, B. WhitNeY ${ }^{7}$, AND A. WOOTTEN ${ }^{8}$ \\ ${ }^{1}$ Department of Physics and Astronomy and Center for Interdisciplinary Research in Astronomy, Northwestern University, Evanston, IL 60208, USA \\ ${ }^{2}$ Department of Physics and Astronomy, and Centre for Astronomy, Astrophysics, \& Astrophotonics, Macquarie University, Sydney, NSW 2109, Australia \\ ${ }^{3}$ CREST/UMBC/NASA GSFC, Code 665, Greenbelt, MD 20771, USA \\ ${ }^{4}$ Space Telescope Science Institute, 3700 San Martin Drive, Baltimore, MD 21218, USA \\ ${ }^{5}$ California Institute of Technology, MC 320-47, Pasadena, CA 91125, USA \\ ${ }^{6}$ Department of Astronomy, University of Maryland, MD 20742, USA \\ ${ }^{7}$ Space Science Institute, 4750 Walnut Street, Suite 205, Boulder, CO 80301, USA \\ ${ }^{8}$ National Radio Astronomy Observatory, Charlottesville, VA 22903, USA \\ Received 2013 February 12; accepted 2013 March 12; published 2013 April 5
}

\begin{abstract}
ALMA observations of the Galactic center with a spatial resolution of $2^{\prime \prime} .61 \times 00^{\prime \prime} 97$ resulted in the detection of $11 \mathrm{SiO}(5-4)$ clumps of molecular gas within $0.6 \mathrm{pc}\left(15^{\prime \prime}\right)$ of Sgr A*, interior to the 2 pc circumnuclear molecular ring. The three $\mathrm{SiO}(5-4)$ clumps closest to $\mathrm{Sgr} \mathrm{A}^{*}$ show the largest central velocities, $\sim 150 \mathrm{~km} \mathrm{~s}^{-1}$, and the broadest asymmetric line widths with full width zero intensity (FWZI) $\sim 110-147 \mathrm{~km} \mathrm{~s}^{-1}$. The remaining clumps, distributed mainly to the NE of the ionized mini-spiral, have narrow FWZI $\left(\sim 18-56 \mathrm{~km} \mathrm{~s}^{-1}\right)$. Using CARMA SiO (2-1) data, Large Velocity Gradient modeling of the SiO line ratios for the broad velocity clumps constrains the column density $\mathrm{N}(\mathrm{SiO}) \sim 10^{14} \mathrm{~cm}^{-2}$, and the $\mathrm{H}_{2}$ gas density $n_{\mathrm{H}_{2}}=(3-9) \times 10^{5} \mathrm{~cm}^{-3}$ for an assumed kinetic temperature $100-200 \mathrm{~K}$. The $\mathrm{SiO}$ clumps are interpreted as highly embedded protostellar outflows, signifying an early stage of massive star formation near Sgr A* in the last $10^{4}-10^{5} \mathrm{yr}$. Support for this interpretation is provided by the $\mathrm{SiO}$ (5-4) line luminosities and velocity widths which lie in the range measured for protostellar outflows in star-forming regions in the Galaxy. Furthermore, spectral energy distribution modeling of stellar sources shows two young stellar object candidates near SiO clumps, supporting in situ star formation near Sgr A*. We discuss the nature of star formation where the gravitational potential of the black hole dominates. In particular, we suggest that external radiative pressure exerted on self-shielded molecular clouds enhances the gas density, before the gas cloud becomes gravitationally unstable near Sgr $\mathrm{A}^{*}$. Alternatively, collisions between clumps in the ring may trigger gravitational collapse.
\end{abstract}

Key words: Galaxy: center - ISM: jets and outflows - ISM: molecules - stars: protostars

Online-only material: color figures

\section{INTRODUCTION}

The compact radio source $\operatorname{Sgr} \mathrm{A}^{*}$, located at the very dynamical center of our Galaxy, coincides with a $4 \times 10^{6} M_{\odot}$ black hole (Ghez et al. 2008; Gillessen et al. 2009). Star formation near Sgr A* is forbidden, unless the gas density is large enough for self-gravity to overcome the strong tidal shear of the back hole. One viable mechanism is cloud capture to form a massive and dense gaseous disk around the black hole. The disk becomes gravitationally unstable, creating a new generation of stars (e.g., Levin \& Beloborodov 2003; Nayakshin et al. 2007; Paumard et al. 2006; Lu et al. 2009; Mapelli et al. 2012; Wardle \& Yusef-Zadeh 2008; Bonnell \& Rice 2008; Alig et al. 2012). This formation scenario has been applied to disk-like distributions of young stars on a scale of $0.03-0.3 \mathrm{pc}$, to the $2-5$ pc circumnuclear molecular ring (CND or CNR) orbiting Sgr A*, as well as to active galactic nuclei with megamaser disks (Wardle \& Yusef-Zadeh 2008, 2012). The Galactic center provides an opportunity for testing models of stellar birth with far reaching implications on the nature of star formation in the nuclei of galaxies hosting massive black holes.

Because the age of the stellar disk orbiting Sgr A* is several million years, it is not possible to identify signatures of early phases of star formation such as maser activity near the black hole. However, a number of recent studies suggest infrared excess sources in the $\mathrm{N}$ arm, as well as young stellar object (YSO) candidates in IRS $13 \mathrm{~N}$ within a projected distance of 0.12 pc (3") from Sgr A* (e.g., Viehmann et al. 2006; Fritz et al. 2010; Eckart et al. 2013; Nishiyama \& Schödel 2013). These measurements imply that star formation has taken place within $\sim 10^{5}$ yr. On a larger scale of $50^{\prime \prime}-100^{\prime \prime}$, or a projected distance of 2-4 pc (assuming that the distance to the Galactic center is $8.5 \mathrm{kpc}$ ), the molecular ring (Jackson et al. 1993; Montero-Castano et al. 2009; Martin et al. 2012) which encircles Sgr $A^{*}$ with a rotational velocity of $\sim 100 \mathrm{~km} \mathrm{~s}^{-1}$ shows signatures of massive star formation activity within the last $\sim 10^{4}-10^{5}$ yr (Yusef-Zadeh et al. 2008). Here, we present the earliest signatures of ongoing star formation on a scale of about $11^{\prime \prime}(0.44 \mathrm{pc})$ from $\mathrm{Sgr} \mathrm{A}^{*}$ : the presence of $\mathrm{SiO}(5-4)$ line emission around the interior of the molecular ring. The $\mathrm{SiO}$ molecule is an excellent tracer of protostellar outflows and is shock excited, since silicon is removed from dust grains, significantly increasing gas-phase abundance.

\section{OBSERVATIONS AND DATA REDUCTION}

ALMA. The data were obtained through the ALMA Science Verification process on 2011 June 26. Utilizing only 12 of the $12 \mathrm{~m}$ antennas, the observations consisted of a 7-point mosaic at the position of Sgr $\mathrm{A}^{*}$ : 
Table 1

Gaussian Line Parameters of Fitted SiO Sources

\begin{tabular}{lcccrr}
\hline \hline Source & $\begin{array}{c}\text { R.A. } \\
(\mathrm{J} 2000)\end{array}$ & $\begin{array}{c}\text { Decl. } \\
(\mathrm{J} 200)\end{array}$ & $\begin{array}{c}\text { Peak } \\
\left(\mathrm{mJy} \mathrm{beam}^{-1}\right)\end{array}$ & $\begin{array}{c}\text { Center } \\
\left(\mathrm{km} \mathrm{s}^{-1}\right)\end{array}$ & $\begin{array}{c}\text { FWHM } \\
\left(\mathrm{km} \mathrm{s}^{-1}\right)\end{array}$ \\
\hline 1 & $17: 45: 40.51$ & -29.0 .28 .78 & $38.91 \pm 2.28$ & $147.81 \pm 1.35$ & $47.42 \pm 3.29$ \\
2 & $17: 45: 40.74$ & -29.0 .26 .11 & $13.92 \pm 1.36$ & $159.81 \pm 2.60$ & $54.81 \pm 6.39$ \\
3 & $17: 45: 40.61$ & -29.0 .23 .90 & $35.43 \pm 1.84$ & $-27.86 \pm 0.69$ & $27.11 \pm 1.66$ \\
4 & $17: 45: 41.01$ & -29.0 .27 .03 & $77.75 \pm 3.17$ & $7.98 \pm 0.23$ & $11.52 \pm 0.55$ \\
5 & $17: 45: 41.06$ & -29.0 .24 .09 & $62.14 \pm 3.19$ & $14.28 \pm 0.28$ & $11.15 \pm 0.66$ \\
6 & $17: 45: 41.17$ & -29.0 .17 .84 & $67.20 \pm 3.64$ & $20.85 \pm 0.34$ & $12.92 \pm 0.81$ \\
7 & $17: 45: 41.03$ & -29.0 .14 .25 & $75.74 \pm 2.80$ & $38.98 \pm 0.26$ & $14.66 \pm 0.63$ \\
8 & $17: 45: 40.83$ & -29.0 .12 .78 & $48.81 \pm 2.80$ & $39.96 \pm 0.44$ & $15.65 \pm 1.05$ \\
9 & $17: 45: 40.03$ & -29.0 .12 .87 & $62.31 \pm 2.53$ & $77.80 \pm 0.38$ & $19.44 \pm 0.93$ \\
10 & $17: 45: 40.31$ & -29.0 .43 .77 & $71.32 \pm 2.75$ & $-42.98 \pm 0.39$ & $21.02 \pm 0.95$ \\
11 & $17: 45: 39.51$ & -29.0 .36 .96 & $50.43 \pm 1.81$ & $136.16 \pm 0.96$ & $55.51 \pm 2.43$ \\
\hline
\end{tabular}

$(\alpha, \delta)=17^{\mathrm{h}} 45^{\mathrm{m}} 40^{\mathrm{s}} .04,-29^{\circ} 0^{\prime} 28^{\prime \prime} .12$. Titan was initially used as the flux calibrator and 3C279 as the initial bandpass and phase calibrator. NRAO 530 was observed periodically to correct for any changes in phase and amplitude as a function of time. Four basebands of total width $1.875 \mathrm{GHz}$ were used with the high spectral resolution, which contained 3840 channels each. The spectral windows were centered at roughly $216.2 \mathrm{GHz}$, 218.0 GHz, 231.9 GHz, and 233.7 GHz. The SiO (5-4) line emission is centered at $217.105 \mathrm{GHz}$. The editing and calibration of the data was carried out by the Science Verification team in CASA. The imaging was completed by modifying the supplied imaging script. SiO 5-4 fell at the intersection of the first two basebands at $216.2 \mathrm{GHz}$ and $218.0 \mathrm{GHZ}$. As a result, care was taken to note any changes in amplitude and phase at the edge channels in combining the two windows. A linear continuum was fitted and subtracted from the line-free channels before CLEANing. The data was averaged by a factor of five to achieve a spectral resolution of $3.371 \mathrm{~km} \mathrm{~s}^{-1}(2.441 \mathrm{MHz})$ using the raw binning of $0.674 \mathrm{~km} \mathrm{~s}^{-1}(0.488 \mathrm{MHz})$. To avoid confusion with nearby lines, we studied velocity structures within $440 \mathrm{~km} \mathrm{~s}^{-1}$ spatial resolutions of $2^{\prime \prime} .61 \times 0$ 0.97.

CARMA. The SiO (2-1) line data were taken with the Combined Array for Research in Millimeter-wave Astronomy (CARMA) during the 2009 and 2010 observing seasons in the $\mathrm{D}$ and $\mathrm{C}$ array configurations. The array consisted of six $10.4 \mathrm{~m}$ antennas and nine $6.1 \mathrm{~m}$ antennas and the maps were made on a 127-point hexagonal mosaic, Nyquist-sampling the $10.4 \mathrm{~m}$ antenna primary beam. The spatial resolution and spectral resolutions of the final maps are $8 . .87 \times 4^{\prime \prime} .56$ and $6.74 \mathrm{~km} \mathrm{~s}^{-1}$, respectively.

\section{RESULTS AND DISCUSSION}

Figure 1(a) shows a composite $3.6 \mathrm{~cm}$ continuum image of the three arms of the mini-spiral (Sgr A West) surrounded by the CNR as traced by HCN line emission (Christopher et al. 2005). The inner $1^{\prime}(2.4 \mathrm{pc})$ of the CNR, as observed with ALMA, shows a large concentration of molecular clumps in the $\mathrm{SiO}$ (5-4) line emission. The distribution of molecular and ionized gas orbiting Sgr A* is generally described as a central cavity that consists of ionized gas of Sgr A West coupled to the surrounding molecular ring (e.g., Roberts \& Goss 1993; Serabyn \& Lacy 1985). A string of SiO clumps is found in the region between the $\mathrm{N}$ and $\mathrm{E}$ arms of Sgr A West. A close up view of these clumps is shown in Figure 1(b). What is most interesting is the presence of $\mathrm{SiO}$ clumps in the interior of the ring, which is expected to be dominated by the central cavity of ionized gas and atomic gas traced by [O I] emission, devoid of any molecular gas. Figure 1(b) also shows two clumps (green), with velocities $v_{\mathrm{r}} \sim 136-160 \mathrm{~km} \mathrm{~s}^{-1}$, and FWHM line widths $47-55 \mathrm{~km} \mathrm{~s}^{-1}$. These ALMA clumps, 1 and 11, are located about $7^{\prime \prime} \mathrm{NE}$ and $11^{\prime \prime} \mathrm{SW}$ of Sgr A*, respectively, and do not follow the kinematics of the molecular ring orbiting Sgr A*. Figure 1(c) shows the positions of these highly redshifted velocity clumps on a 3.6 $\mu \mathrm{m}$ image taken with the Very Large Telescope (VLT). Clumps 1 and 2 lie $\sim 2^{\prime \prime}$ west of IRS $1 \mathrm{~W}$, a mass-losing W-R star with a bow shock structure (Tanner et al. 2005).

To illustrate the kinematics of $\mathrm{SiO}$ (5-4) molecular clumps, the spectra of the broadest velocity clumps are shown in Figure 1(d) whereas the spectra of eight clumps with narrow line widths within the ring are shown in Figure 2 where the distribution of $\mathrm{SiO}(5-4)$ peak line emission is shown. Columns 1-7 of Table 1 list the source numbers, coordinates, intensity of the peak emission, Gaussian fitted central velocity, FWHM line widths, and full width zero intensity (FWZI) of the 11 sources labeled in Figure 2. FWZI line widths are listed because many sources show velocity profiles with blueshifted wings (e.g., Clumps 1,2,11). Clumps 4-8 run parallel to the eastern edge of the $\mathrm{N}$ arm of ionized gas showing typical line widths between 11 and $21 \mathrm{~km} \mathrm{~s}^{-1}$ and peak radial velocities that decrease from $37.8 \mathrm{~km} \mathrm{~s}^{-1}$ at Clump 8 to $7.4 \mathrm{~km} \mathrm{~s}^{-1}$ at Clump 4. The trend in radial velocity change in molecular clumps is consistent with the trend in the kinematics of ionized gas of the $\mathrm{N}$ arm (e.g., Zhao et al. 2010). However, the central velocities of the ionized gas in the $\mathrm{N}$ arm are $\sim 100 \mathrm{~km} \mathrm{~s}^{-1}$ and decrease to $0 \mathrm{~km} \mathrm{~s}^{-1}$ as the ionized gas approaches closer to Sgr A* (e.g., Roberts \& Goss 1993; Jackson et al. 1993; Zhao et al. 2010). The kinematics of $\mathrm{SiO}(5-4)$ is dissimilar to that of the ionized gas of the $\mathrm{N}$ arm and the "tongue" of neutral [O I] gas. The spatial and velocity distributions of $\mathrm{SiO}(5-4)$ suggest a clumpy and dense molecular cloud lying in the interior of the molecular ring.

Clumps 1-3 defy the kinematical trend of the $\mathrm{N}$ arm: they display highly redshifted $\left(<160 \mathrm{~km} \mathrm{~s}^{-1}\right)$ and blueshifted velocity $\left(-28 \mathrm{~km} \mathrm{~s}^{-1}\right)$ components and lie adjacent to the ionized gas of the $\mathrm{N}$ arm, but with dissimilar velocities. Figure 3(a) shows contours of highly redshifted $\mathrm{SiO}$ (5-4) line emission from Clumps 1 and 2 superimposed on a $3 \mathrm{~cm}$ continuum image of the $\mathrm{N}$ arm. The SiO line emission from Clump 1 appears to be elongated to the NE with non-Gaussian velocity profiles having blueshifted wings. Another morphological structure of the $\mathrm{N}$ arm is the wavy structure along the direction of the flow (YusefZadeh \& Wardle 1993; Zhao et al. 2010). Figure 3(b) shows the contours of $\mathrm{SiO}(5-4)$ emission from Clump 3 superimposed on a $3 \mathrm{~cm}$ continuum image. The spectrum of Clump 3 that lies 


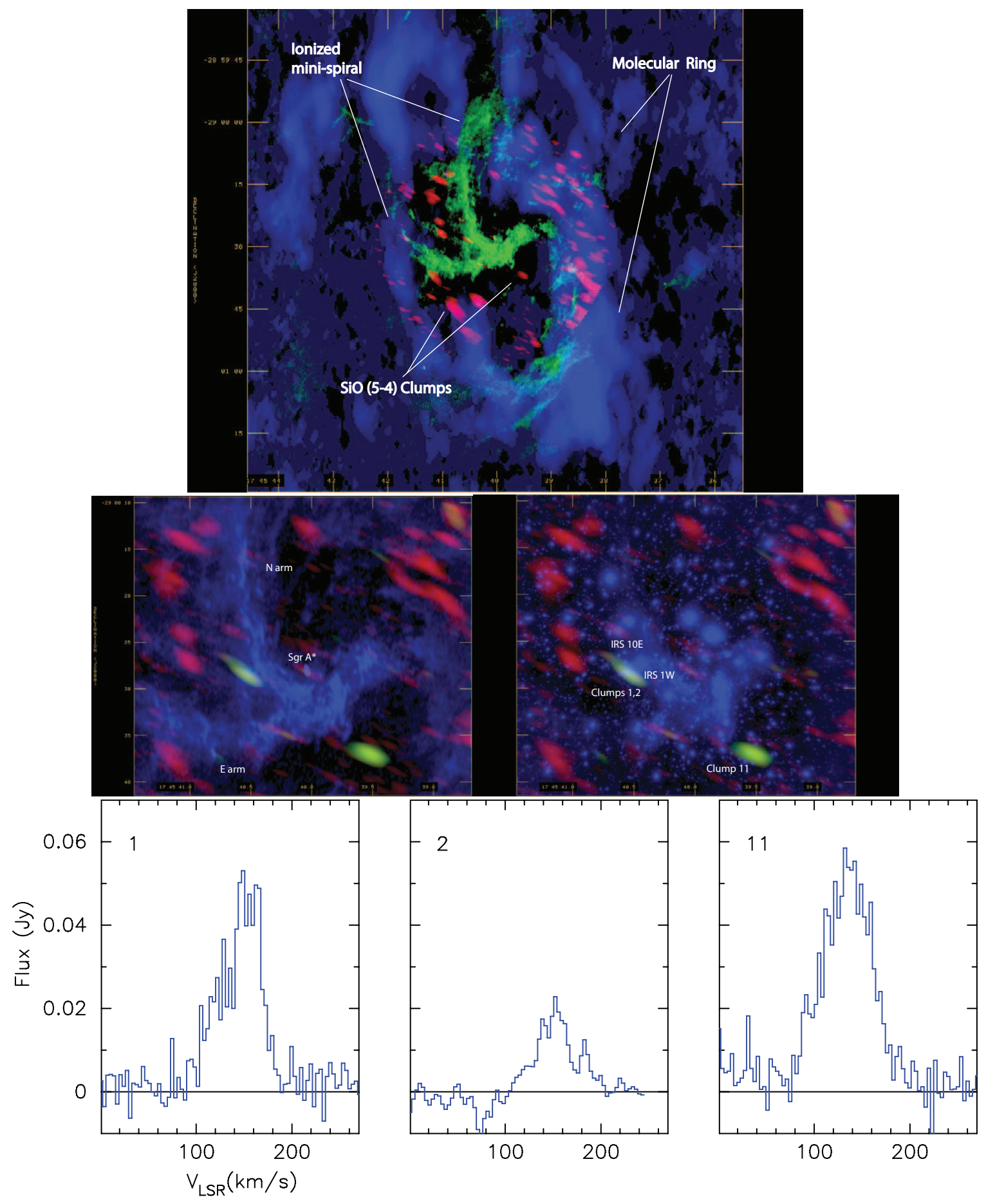

Figure 1. (a) A composite image of the inner $2^{\prime}$ of the Galactic center: $3.6 \mathrm{~cm}$ continuum image of Sgr A West (green), HCN (1-0) emission from the molecular ring (blue; Christopher et al. 2005), and SiO (5-4) line emission from the central region of the ring (red). (b) The distribution of the SiO line emission (red) integrated over velocities $150<v<200 \mathrm{~km} \mathrm{~s}^{-1}$ and superimposed on a $3.6 \mathrm{~cm}$ continuum image (blue). The edge of the SiO (5-4) clump distribution is noisier because of shorter integration time and is limited by the size of the region mapped by ALMA. The highest redshifted velocity SiO (5-4) Clumps 1 and 11 are shown in green. (c) Similar to (b) except that a $3.6 \mu \mathrm{m}$ image taken with the VLT in blue replaces the $3.6 \mathrm{~cm}$ image. (d) The spectra of Clumps 1,2, and 11.

(A color version of this figure is available in the online journal.)

adjacent to the wavy structure shows a radial velocity peaking at $v \sim-28 \mathrm{~km} \mathrm{~s}^{-1}$ and line width of $v_{\mathrm{FWHM}} \sim 27 \mathrm{~km} \mathrm{~s}^{-1}$ with a broad blueshifted wing extending to $-100 \mathrm{~km} \mathrm{~s}^{-1}$. This radial velocity is inconsistent with circular motion orbiting Sgr A*. Several radio dark clouds (Yusef-Zadeh 2012) lie to the east of the $\mathrm{N}$ arm in Figures 3(a) and (b) where Clumps 1-3 lie. These dark features trace molecular gas and are embedded in the ionized gas of the $\mathrm{N}$ arm. Although the morphology of thermal radio emission at the location of Clumps 1 and 3 suggests that the flow of ionized gas is distorted as a result of its interactions with molecular clumps, the kinematics of the molecular and ionized gas are inconsistent with the interaction picture.

Protostellar outflows. To examine the physical properties of $\mathrm{SiO}$ clumps, we focus on the $\mathrm{SiO}(5-4)$ and (2-1) line profiles, as shown in Figure 3(c), with a peak line emission $T_{\mathrm{MB}} \sim 0.95$ and $0.65 \mathrm{~K}$ for Clump 1 and $T_{\mathrm{MB}} \sim 1.1$ and $1 \mathrm{~K}$ for Clump 11 

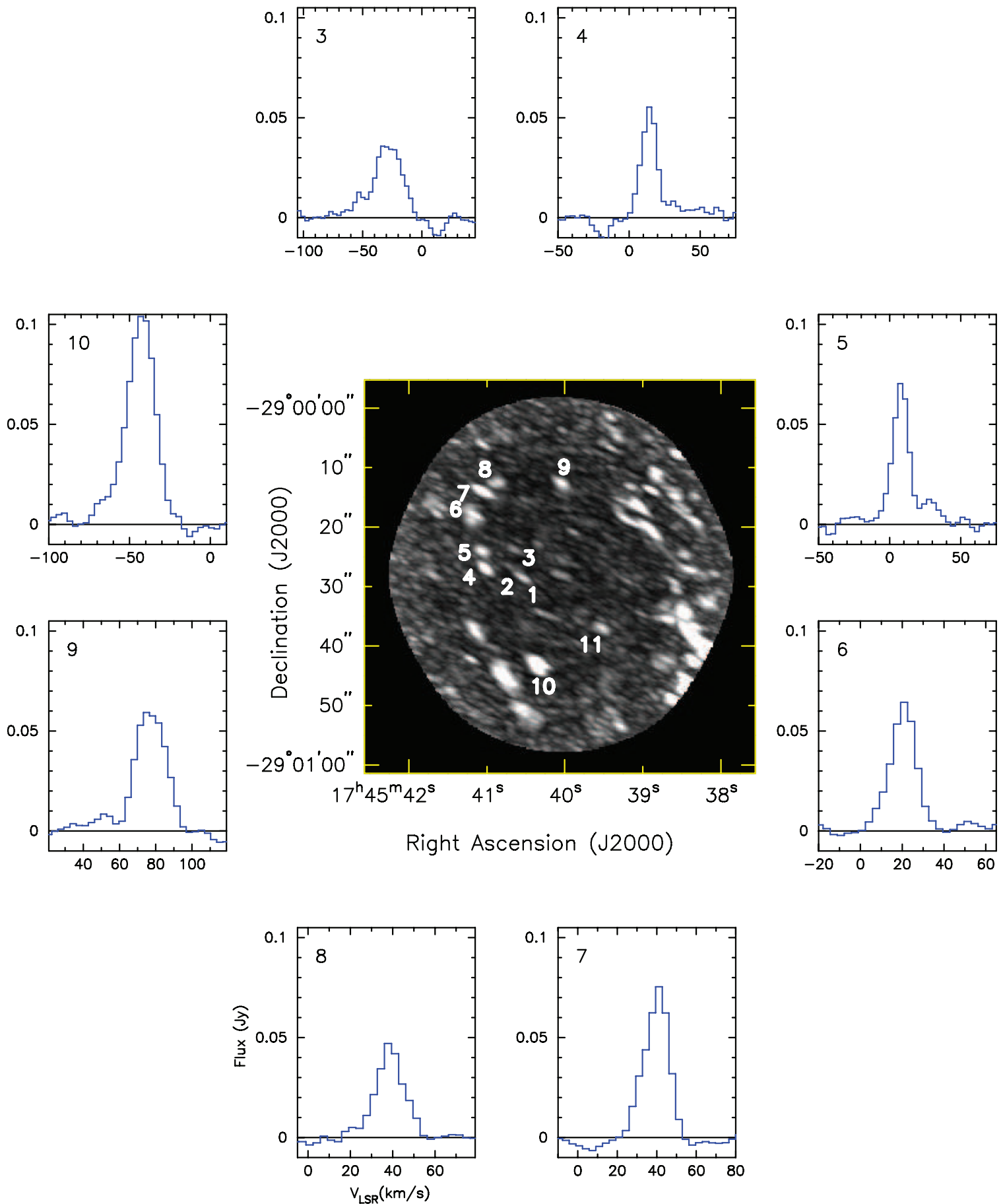

Figure 2. Grayscale image constructed from the peak $\mathrm{SiO}(5-4)$ line emission between $-191<v<213 \mathrm{~km} \mathrm{~s}^{-1}$. The inset shows the spectra of eight $\mathrm{SiO}$ (5-4) clumps (Jy on $Y$-axis vs. $\mathrm{km} \mathrm{s}^{-1}$ on the $X$-axis) within the molecular ring. The positions of the labeled spectra are listed in Table 1.

(A color version of this figure is available in the online journal.)

at a central velocity of $\sim 147$ and $\sim 136 \mathrm{~km} \mathrm{~s}^{-1}$, respectively. The similarity of the $\mathrm{SiO}(5-4)$ and (2-1) line profiles toward Clumps 1 and 11 is a classic signature of one-sided molecular outflows in star-forming regions (e.g., Plambeck \& Menten 1990). We used the Large Velocity Gradient (LVG) model to constrain the density and column density. The result of this analysis is shown in Figure 3(d). Assuming that the beam filling factor is 0.5 , thus $1 \mathrm{mJy}$ is equivalent to $T_{\mathrm{MB}}=20.5$ and $7.96 \mathrm{~m} \mathrm{~K}$ for $\mathrm{SiO}(5-4)$ and $\mathrm{SiO}(2-1)$ data, respectively, and the gas density of hydrogen nuclei is constrained to $n_{\mathrm{H}_{2}} \sim(3-9) \times 10^{5} \mathrm{~cm}^{-3}$ within a temperature range of
$100-200 \mathrm{~K}$. The synthesized beam is highly elongated in one direction, thus we chose a beam filling factor of 0.5. Multitransition $\mathrm{CO}$ and $\mathrm{NH}_{3}$ studies of the inner 2 pc derive kinetic temperatures of 100-200 K and warm gas in the interior of the molecular ring (Bradford et al. 2005; Herrnstein \& Ho 2005). $\mathrm{N}(\mathrm{SiO}) / \Delta v \sim 1.7-2.4 \times 10^{12} \mathrm{~cm}^{-2} \mathrm{~km} \mathrm{~s}^{-1}$ is better constrained than the gas density, as shown in the bottom panel of Figure 3(d). The column densities of $\mathrm{SiO}$ for Clumps 1 and 11 are estimated to be $\mathrm{N}(\mathrm{SiO}) \sim 8.6 \times 10^{13}$ and $\sim 1.3 \times 10^{14} \mathrm{~cm}^{-2}$, respectively. The total mass of molecular gas is estimated to be $\sim 0.2 M_{\odot}$ using a clump radius of $0.03 \mathrm{pc}\left(0^{\prime \prime} 79\right)$ for 

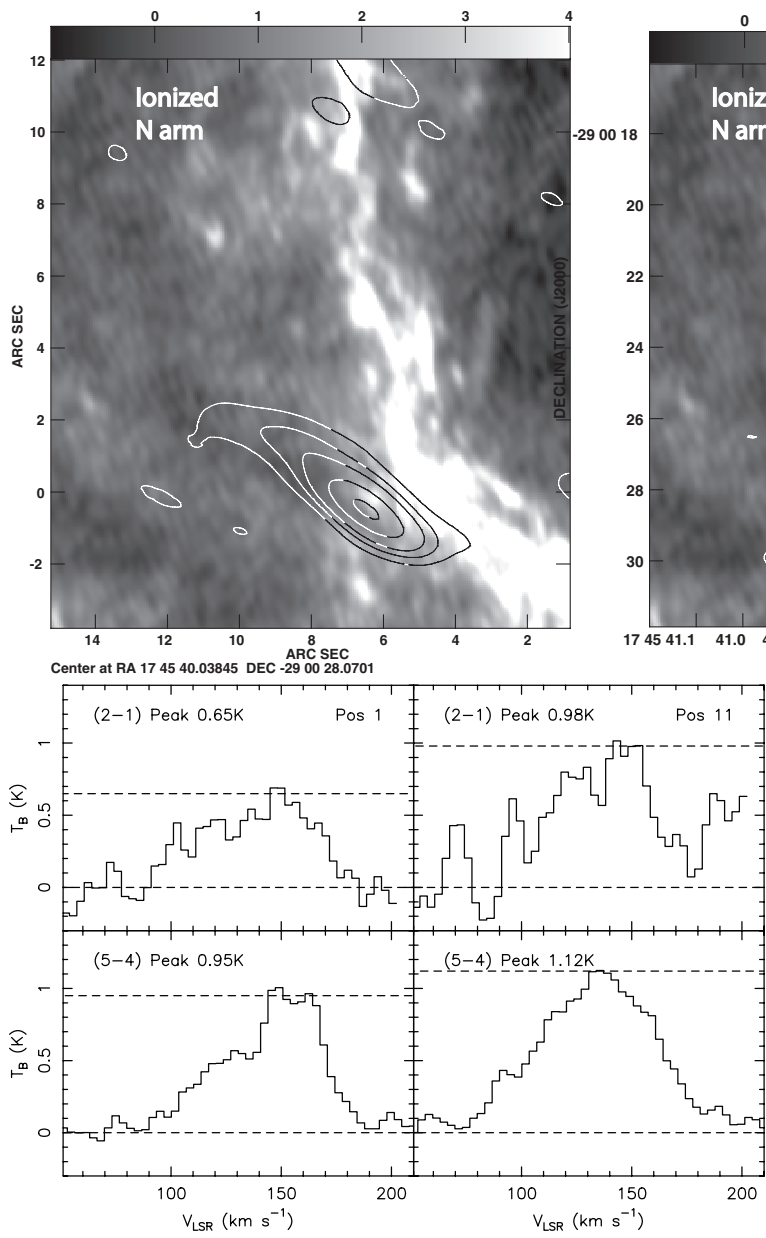
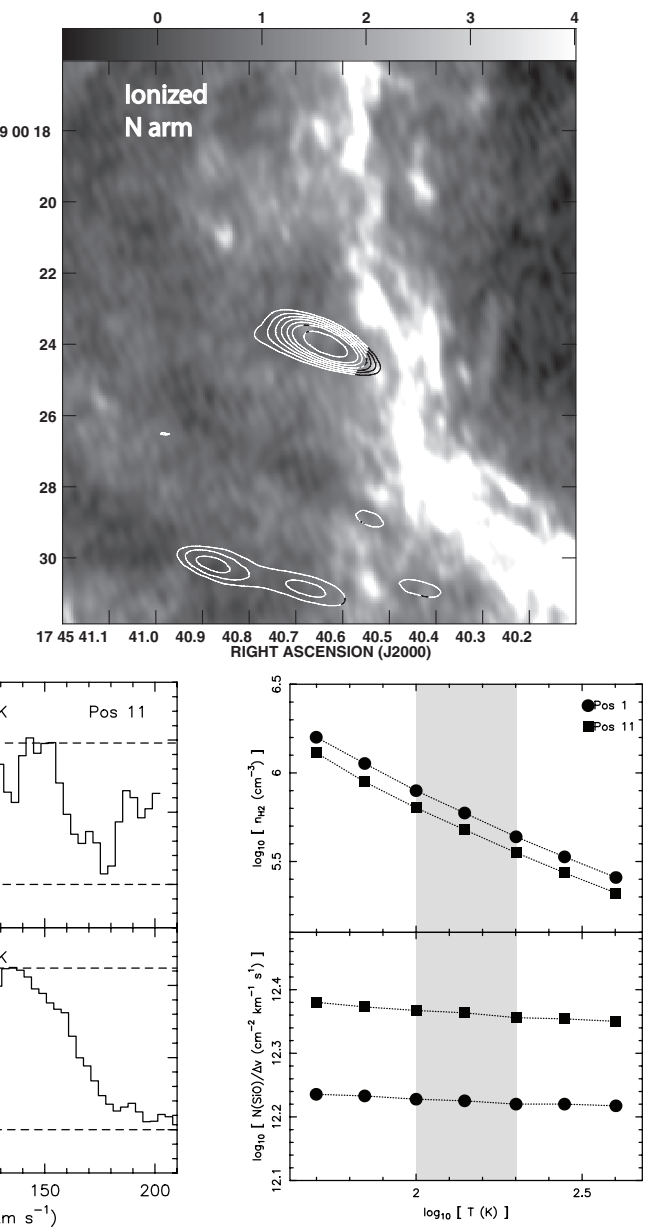
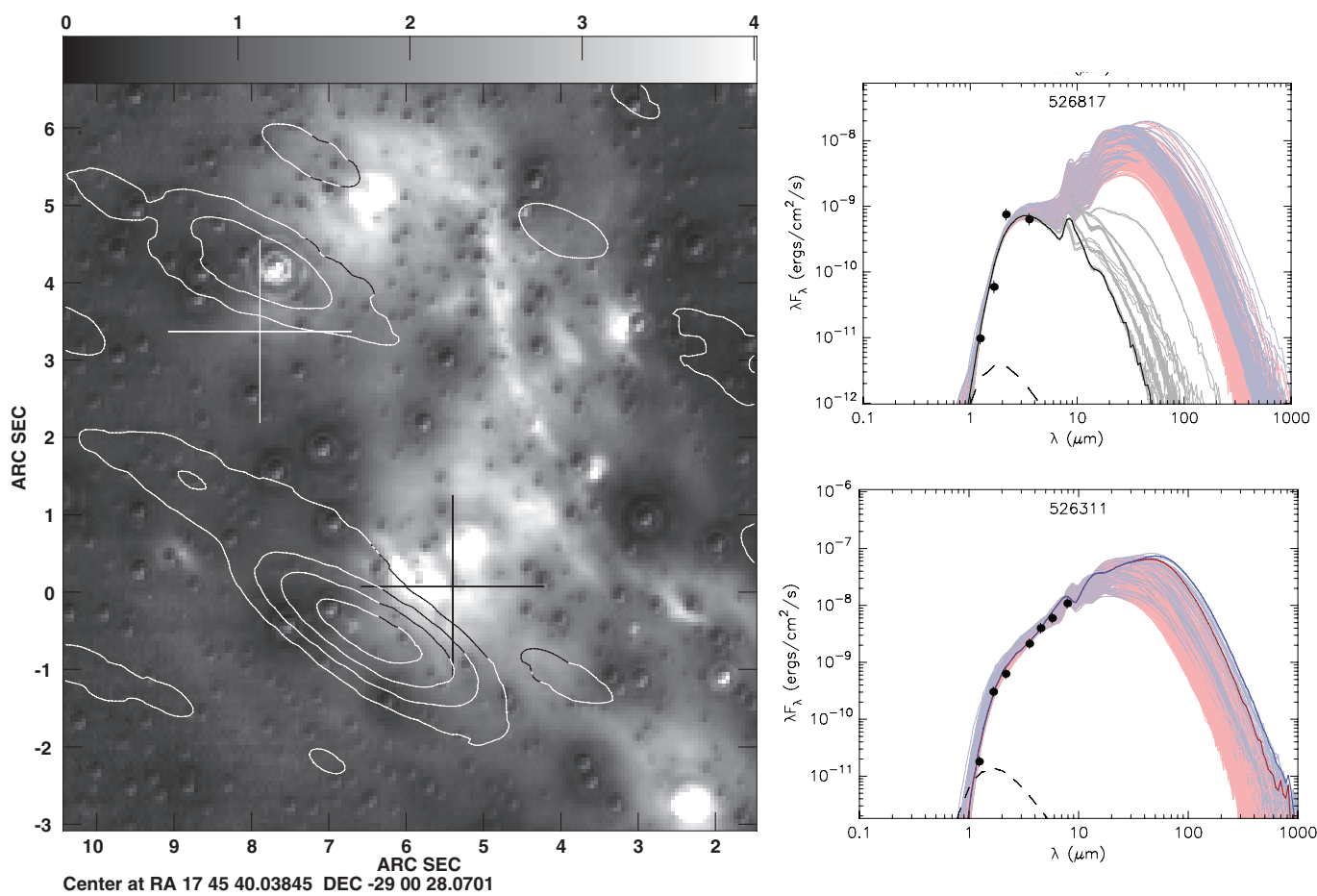

Figure 3. (a) Contours of $\mathrm{SiO}(5-4)$ line emission from Clumps 1 and 2 integrated between 88 and $188 \mathrm{~km} \mathrm{~s}^{-1}$ superimposed on a $3 \mathrm{~cm}$ continuum image. (b) Same as (a) except contours of $\mathrm{SiO}$ (5-4) line emission integrated over 0 and $-50 \mathrm{~km} \mathrm{~s}^{-1}$. (c) $\mathrm{SiO}(5-4)$ and (2-1) line profiles of Clumps 1 and 11 (labeled as POS 1 and 11). (d) The inferred $\mathrm{H}_{2}$ density (top) and $\mathrm{N}(\mathrm{SiO}) / \Delta v$ (bottom) as a function of temperature. The band (gray) shows the temperature range of the CNR. (e) Contours of $\mathrm{SiO}(5-4)$ emission from Clumps 1 and 3 superimposed on a ratio map of $L(3.6 \mu \mathrm{m})$ to $K(1.6 \mu \mathrm{m})$ bands. The crosses show the positions of YSO candidates. (f) Fitted SEDs of the two YSO candidates 526311 and 526817 in the vicinity of Clumps 1 and 3, respectively. 526311 and 526817 are designated in the catalog by Ramirez et al. (2008).

(A color version of this figure is available in the online journal.) 


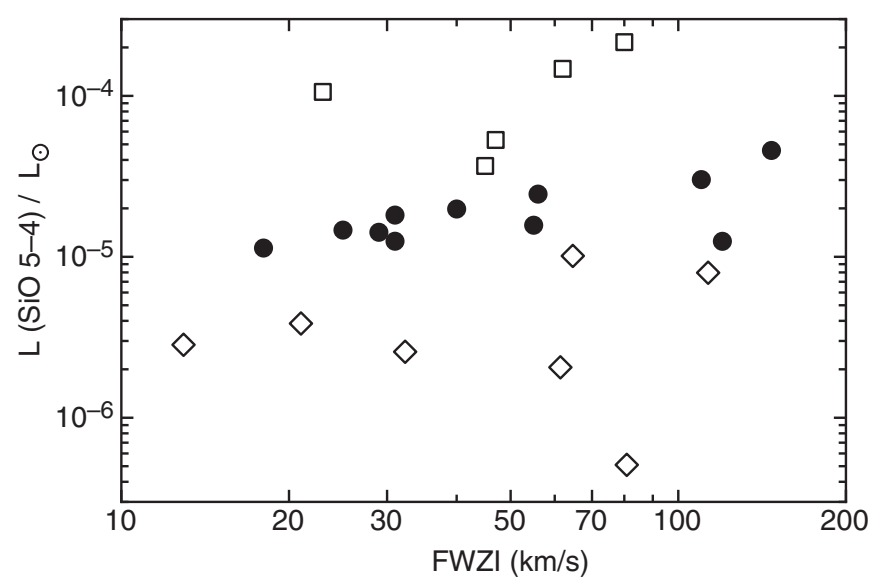

Figure 4. Filled circles show the luminosity in the $\mathrm{SiO}(5-4)$ line vs. FWZI for the 11 sources detected in the circumnuclear ring. The open diamonds and open squares show the corresponding quantities for outflows from low-mass YSOs (Gibb et al. 2004) and high-mass YSOs (Gibb et al. 2007), respectively.

unresolved sources and $n_{\mathrm{H}_{2}}=10^{6} \mathrm{~cm}^{-3}$. This corresponds to a column density of $1.8 \times 10^{23} \mathrm{~cm}^{-2}$ and $\mathrm{SiO}$ abundance of $5.6 \times 10^{-10}$ which is clearly much higher than is expected in quiescent molecular clouds. Using the size and the FWHM velocity widths, the velocity gradient is estimated to be 833 and $165 \mathrm{~km} \mathrm{~s}^{-1} \mathrm{pc}^{-1}$ for clumps with broad $\left(\sim 50 \mathrm{~km} \mathrm{~s}^{-1}\right)$ and narrow $\left(\sim 10 \mathrm{~km} \mathrm{~s}^{-1}\right)$ line widths, respectively. These imply that the clumps cannot be bound by self-gravity because the collapse timescale is $12-58$ times longer than the dynamical timescales $t_{\text {dyn }} \sim(0.3-1.5) \times 10^{3} \mathrm{yr}$, and thus are likely to be outflows from YSOs. The blueshifted velocity wings and non-circular radial velocities in the orbital motion about $\mathrm{Sgr} \mathrm{A}^{*}$ is also consistent with $\mathrm{SiO}$ outflows in which the approaching side of the outflow has burst through the edge of a molecular cloud.

One strong piece of evidence that the $\mathrm{SiO}$ sources are YSO outflows is that the luminosities and velocity widths lie in the range detected from protostellar outflows in star-forming regions. Figure 4 compares the $\mathrm{SiO}(5-4)$ luminosity and FWZI for the detected sources inside the molecular ring with those for samples of low-mass and high-mass YSOs (Gibb et al. 2004, 2007). The SiO 5-4 luminosities for the low-mass and highmass YSO samples tabulated in Gibb et al. $(2004,2007)$ have been corrected by multiplying by 1500 and 170, respectively (A. Gibb 2013, private communication). All three samples show the same wide range of velocity widths, and the luminosities of $\mathrm{SiO}$ clumps interior to the molecular ring fall in between those of the low-mass and high-mass YSOs.

What sources drive the outflows? We identified two new YSO candidates in the $\mathrm{N}$ arm near Clumps 1 and 3. Figure 3(e) shows contours of $\mathrm{SiO}(5-4)$ emission from Clumps 1 and 5 superimposed on a ratio map of $L(3.6 \mu \mathrm{m})$ to $K(1.6 \mu \mathrm{m})$ band images from VLT observations. The ratio map shows the dusty environment of the $\mathrm{N}$ arm. The crosses coincide with the positions of YSO candidates with the positional uncertainty of $1^{\prime \prime} .18$ at $8 \mu \mathrm{m}$ (Ramirez et al. 2008). The spectral energy distributions (SEDs) of these sources are analyzed by comparing a set of SEDs enhanced by a large grid of YSO models (Whitney et al. 2003; Robitaille et al. 2007), as Figure 3(f) shows their fitted SEDs. The source 526311, which is selected from their IR colors, is classified as a Stage I YSO candidate, whereas the red source 526817 is a YSO candidate but its classification is uncertain. These YSO candidates have typical ages of $10^{5}$ yr. By fitting the SEDs, we derive masses of $34.3 \pm 5.9$ and
$19.4 \pm 2.5 M_{\odot}$, luminosities $1.8 \pm 0.7 \times 10^{5}$ and $4.4 \pm 1.5 \times 10^{4} L_{\odot}$ and mass-loss rates $5.1 \pm 0.1 \times 10^{-4}$ and $2.5 \pm 0.1 \times 10^{-4} M_{\odot} \mathrm{yr}^{-1}$ for Clumps 1 and 5, respectively. YSO candidate 526817 coincides with the brightest source IRS 10E (Viehmann et al. 2006) at the center of Clump 3 whereas the YSO candidate 526817 is an unresolved component of multiple sources in IRS 1. The proximity of massive YSO candidates, SiO (5-4) Clumps near IRS 1 and IRS 10 clusters containing W-R stars, suggest that there is still on-going star formation near these stellar clusters. Similar distributions of massive YSOs, and W-R stars are found in the IRS 13 cluster (Fritz et al. 2010; Eckart et al. 2013).

Star formation mechanism. We have detected the presence of several $\mathrm{SiO}(5-4)$ clumps within a pc of Sgr A*. Their $\mathrm{SiO}$ (5-4) luminosities, non-Gaussian velocity profiles, and large line widths unbound by self-gravity and dense gas, as traced by dark radio clouds, all point to the conclusion that these clumps are tracing YSOs with protostellar outflows. Thus, our observations reveal the earliest stages of massive star formation near Sgr A* on a timescale of $\sim 10^{4}-10^{5} \mathrm{yr}$. Additional support for star formation on a timescales of $\sim 10^{5} \mathrm{yr}$ come from SED fitted YSO candidates with infrared excesses, as well as young stellar disks orbiting Sgr A*, respectively. These suggest that star formation is continuous near Sgr $\mathrm{A}^{*}$.

The mechanism by which star formation can take place in this tidally stressed environment is not well understood. The $\mathrm{H}_{2}$ density in the molecular ring, $\sim 10^{6}-10^{7} \mathrm{~cm}^{-3}$, is well below that needed for self-gravity to overcome the tidal field of the central black hole, i.e., the Roche density, $3.4 \mathrm{M} / \mathrm{r}^{3}$, is equivalent to $n(\mathrm{~Hz})=2 \times 10^{8}(\mathrm{r} / \mathrm{pc})^{-3} \mathrm{~cm}^{-3}$. Star formation in this region must therefore be triggered by significant compression of the ambient gas in the ring. Tidal squeezing of an elongated infalling cloud can compress the gas in two dimensions, but the density must be increased by two orders of magnitude as the cloud approaches Sgr A*. A plausible mechanism for production of high density, self-gravitating molecular clumps is through the formation of a gaseous disk in the context of cloud capture by $\mathrm{Sgr} \mathrm{A}^{*}$. In this scenario, the $\mathrm{SiO}$ clumps are predicted to be distributed on a gaseous disk that is captured $10^{4-5}$ years ago and is currently forming stars as it orbits Sgr A* Here we consider two other possibilities: (1) compression by the intense UV radiation field in the Galactic center, and (2) clump-clump collisions.

Hot stars in the central parsec produce an intense radiation field with an effective temperature $\sim 3 \times 10^{4} \mathrm{~K}$ and a luminosity of the order of $\sim 10^{7.5} L_{\odot}$ (Lacy et al. 1980; Davidson et al. 1992). The radiation pressure is capable of producing significant compression. Equating the radiation pressure to the thermal pressure of the compressed molecular gas, i.e., $L /\left(4 \pi c D^{2}\right)=1.2 n_{\mathrm{H}_{2}} k T$, where $D$ is the distance to the center of the hot star distribution, yields $n_{\mathrm{H}_{2}} \sim$ $4 \times 10^{8}\left(L / 10^{7.5} L_{\odot}\right)(D / 0.1 \mathrm{pc})^{-2}(T / 100 \mathrm{~K})^{-1} \mathrm{~cm}^{-3}$, comparable to the critical density. It is therefore possible for the pressure associated with irradiation by the UV field to compress a gas clump to the point of gravitational collapse. Note, however, that the clump must be exposed to the radiation field for about $5 \times 10^{4} \mathrm{yr}$ for the compression to work its way through the entire clump and that much of the momentum may be deposited in a photoevaporative outflow.

Clump-clump collisions are an alternative method for producing $\mathrm{SiO}$ emission, either through the destruction of dust grains in large scale shock waves associated with the collision, or via outflows from YSOs formed, because the compression associated with the shock waves triggered star 
formation. This model, however, has difficulty producing the number of detected sources. Suppose a clump in the interior to the ring has radius $r$, clump-clump velocity dispersion $\mathrm{v}$ and volume filling factor $f$. Then the number of clumps per unit volume is $n_{\mathrm{cl}}=f /\left(4 / 3 \pi r^{3}\right)$, the cross section for almost head-on collisions is $\sigma \sim 2 \pi r^{2}$, and the timescale for a given clump to collide with another is $1 /\left(n_{\mathrm{cl}} \sigma v\right)$. For an ensemble of $\mathrm{N}$ clumps, the number of collisions per unit time is $N n_{\mathrm{cl}} \sigma v \approx 1.5 \times N f v / r$. The collisional interaction time is approximately $2 r / v$, so the number of collisions occurring at any given time is $(N f v / r) \times(2 r / v)=3 f N$. For the interior of the molecular ring with a radius of $15^{\prime \prime}$, a clump size $1^{\prime \prime} .5$, $f=10^{3} / N$ and the number of visible clumps is $3 N f=11$, thus a population of 60 clumps are needed to produce the number of observed $\mathrm{SiO}$ clumps.

In conclusion, ALMA observations show that the interior of the circumnuclear molecular ring is not completely filled with ionized gas (see the review by Genzel et al. 2010) but is a site of molecular clumps and on-going star formation. The line widths of these clumps are too large to be gravitationally bound, thus suggesting outflows from YSOs. The $\mathrm{SiO}$ clumps are interpreted as highly embedded protostellar outflows that are highly excited, showing properties that are similar to those found in Galactic star formation regions. We suggest that the required high gas density is produced by the strong external radiation field from young massive stars compressing the gas, thus inducing star formation or that clump collisions can account for compressing the gas. Future observations will determine the total mass of molecular gas residing inside the ring, will allow estimating the efficiency of star formation within a pc of Sgr $\mathrm{A}^{*}$, and will examine if the molecular gas inside the ring is dynamically important in perturbing the dynamics of stars close to Sgr $\mathrm{A}^{*}$.

We thank Stefan Gillessen for providing us with VLT images. This work is partially supported by grants AST-0807400 from the NSF. This paper makes use of the following ALMA data:

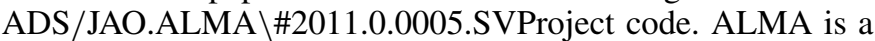
partnership of ESO (representing its member states), NSF (USA), and NINS (Japan), together with the NRC (Canada) and NSC and ASIAA (Taiwan), in cooperation with Chile. The
Joint ALMA Observatory is operated by ESO, AUI/NRAO, and NAOJ.

\section{REFERENCES}

Alig, C., Burkert, A., Johansson, P. H., \& Schartmann, M. 2012, MNRAS, 412, 486

Bonnell, I. A., \& Rice, W. K. M. 2008, Sci, 321, 1060

Bradford, C. M., Stacey, G. J., Nikola, T., et al. 2005, ApJ, 623, 866

Christopher, M. H., Scoville, N. Z., Stolovy, S. R., \& Yun, M. S. 2005, ApJ, 622,346

Davidson, J. A., Werner, M. W., Wu, X., et al. 1992, ApJ, 387, 189

Eckart, A., Muzic, K., Yazici, S., \& Shahzamanian, B. 2013, A\&A, 551, 18

Fritz, T. K., Gillessen, S., Dodds-Eden, K., et al. 2010, ApJ, 721, 395

Genzel, R., Eisenhauer, F., \& Gillessen, S. 2010, RvMP, 82, 3121

Ghez, A. M., Salim, S., Weinberg, N. N., et al. 2008, ApJ, 689, 1044

Gibb, A. G., Davis, C. J., \& Moore, T. J. T. 2007, MNRAS, 382, 1212

Gibb, A. G., Richer, J. S., Chandler, C. J., \& Davis, C. J. 2004, ApJ, 603, 198

Gillessen, S., Eisenhauer, F., Trippe, S., et al. 2009, ApJ, 692, 1075

Herrnstein, R. M., \& Ho, P. T. P. 2005, ApJ, 620, 287

Jackson, J. M., Geis, N., Genzel, R., Harris, A. I., \& Madden, S. 1993, ApJ, 402, 173

Lacy, J. H., Townes, C. H., Geballe, T. R., et al. 1980, ApJ, 241, 132

Levin, Y., \& Beloborodov, A. M. 2003, ApJL, 590, L33

Lu, J. R., Ghez, A. M., Hornstein, S. D., et al. 2009, ApJ, 690, 1463

Mapelli, M., Hayfield, T., Mayer, L., \& Wadsley, J. 2012, ApJ, 749, 168

Martin, M., Martin-Pintado, J., Montero-Castano, M., et al. 2012, A\&A, 539,29

Montero-Castano, M., Herrnstein, R. M., \& Ho, P. T. P. 2009, ApJ, 695, 1477

Nayakshin, S., Cuadra, J., \& Springel, V. 2007, MNRAS, 379, 21

Nishiyama, S., \& Schödel, R. 2013, A\&A, 549, 16

Paumard, T., Genzel, R., Martins, F., et al. 2006, ApJ, 643, 1011

Plambeck, R. L., \& Menten, K. M. 1990, ApJ, 364, 555

Ramirez, S. V., Arendt, R. G., Sellgren, K., et al. 2008, ApJS, 175, 147

Roberts, D. A., \& Goss, W. M. 1993, ApJS, 86, 133

Robitaille, T. P., Whitney, B. A., Indebetouw, R., et al. 2007, ApJS, 169, 328

Serabyn, E., \& Lacy, J. H. 1985, ApJ, 293, 445

Tanner, A., Ghez, A. M., Morris, M. R., \& Christou, J. C. 2005, ApJ, 624,742

Viehmann, T., Eckart, A., Schod̈el, et al. 2006, ApJ, 642, 861

Wardle, M., \& Yusef-Zadeh, F. 2008, ApJL, 683, L37

Wardle, M., \& Yusef-Zadeh, F. 2012, ApJL, 750, L38

Whitney, B. A., Wood, K., Bjorkman, J. E., \& Wolff, M. J. 2003, ApJ, 591, 1049

Yusef-Zadeh, F. 2012, ApJL, 759, L11

Yusef-Zadeh, F., Braatz, J., Wardle, M., \& Roberts, D. 2008, ApJL, 683, L147

Yusef-Zadeh, F., \& Wardle, M. 1993, ApJ, 405, 584

Zhao, J.-H., Blundell, R., Moran, J., et al. 2010, ApJ, 723, 1097 